\title{
Bimanual Pars Plana Vitrectomy for Diabetic Tractional Retinal Detachment Ali A Ghali
}

\author{
Department of Ophthalmology, Faculty of Medicine, Al-Azhar University, Damietta, Egypt
} Corresponding author: Ali A Ghali, Email: ghalyegy@gmail.com, Phone: +20)1152028335

\begin{abstract}
Background: Diabetic retinopathy (DR) is considered the commonest cause of diabetic epiretinal membrane (ERM) worldwide. DR remains one of the leading causes of legal blindness in the working- age population.

Aim of the work: was to evaluate the efficacy, safety and complications of bimanual pars plana vitrectomy (PPV) for management of ERM associated with tractional retinal detachment (TRD).

Patients and methods: 27 eyes in 23 patients presented with diabetic ERM associated with TRD. After full ophthalmic examination all patients underwent bimanual PPV with silicone oil injection or gas tamponade without or with phacoemulsification and posterior chamber intraocular lens (IOL) implantation.

Results: Complete removal of ERM was achieved in 23 cases $(85.18 \%)$ while has incompletely removed in four cases $(14.81 \%)$. Residual tractional RD was observed in one cases (3.7\%). Iatrogenic breaks were occurred in five cases $(18.51 \%)$. Endo-laser photocoagulation was done in 19 cases $(70.73 \%)$. There was improvement of BCVA in $23(85.18 \%)$ cases, while remained the same in two cases $(7.4 \%)$ and deteriorated in two eyes $(7.4 \%)$ due to reproliferation and development of neovascular glaucoma.

Conclusion: It could be concluded that bimanual PPV is an effective, safe and an attainable technique for management of diabetic ERM associated with TRD, learning curve with this technique and coast benefit ratio may be considered.
\end{abstract}

Keywords: bimanual vitrectomy, tractional, retinal detachment.

\section{INTRODUCTION}

Diabetic retinopathy (DR) is considered the commonest cause of ERM worldwide. Despite all the important progress in the understanding and management of diabetes over the recent years, DR remains one of the leading causes of legal blindness in the working- age population ${ }^{(1)}$. The prevalence of DR in diabetic patients was reported as high as 54\% after 10-19 years of diabetes but TRD is the severest stage of proliferative DR (PDR) ${ }^{(2)}$.

Epi-retinal membrane (ERM) is a retinal, fine, semi-translucent, fibro-cellular membrane on the inner retinal surface along the internal limiting membrane (ILM) resulting in visual impairment of variable degrees ${ }^{(3)}$. The origin of this proliferation may be attributed to retinal glial cells, fibrous astrocytes, and Muller cells migrating from neurosensory retina, and composed of macrophages and retinal pigment epithelial (RPE) cells ${ }^{(4)}$.The clinical importance of ERM is the tendency to contract, and pulls the underlying retina due to vitreoretinal adhesions which often results in distortion or TRD ${ }^{(5)}$.The tangential contractile force of ERM is the main factor influencing early vision loss ${ }^{(6)}$.

PPV with membrane peeling is the standard treatment, Surgical outcomes are better in patients with recently reduced vision and poorer in patients with longstanding macular detachment ${ }^{(7,8,9)}$. The results have improved following the advent of better surgical techniques and tools. However, visual prognosis is influenced by many factors including the patient age, location and extent of the TRD, and the duration of macular detachment. Older age, anterior segment neovascularization, longstanding macular detachment have been associated with poor visual outcome ${ }^{(10)}$. However, early vitrectomy and ERM peeling is a controversial issue in patients with good visual acuity, because it may increase the risk of postoperative visual loss and development of cataract (11). Precise sectioning and removal of fibro-vascular membranes is essential step which considered the most critical and challenging event that may be associated with severe complications ${ }^{(12)}$. These complicated situations encountered intraoperatively can be better mastered using bimanual techniques than with conventional single-handed methods ${ }^{(13)}$.

The use of the chandelier illumination system as the source of endo-illumination for bimanual vitrectomy is an essential part of modern minimal incision vitreoretinal surgery, as it can provide reliable fundus view including peripheral vitreous for bimanual surgery (14) .The general concept of bimanual surgery is to decrease the unwanted pull and push-out forces of scissors and pics that are produced on the retina during dissection of the ERMs. It is used in difficult cases of diabetic TRD for the complete and safe removal of ERM as the surgeon can operate with two active hands and apply counteraction ${ }^{(15)}$. The surgeon can apply laser photocoagulation more easily with the use of chandelier and up to the ora serrata with the help of self- indentation, and compatible with the use of wide-angle viewing systems during vitreous surgery ${ }^{(13,16)}$. Another use of chandelier in diabetic retinal surgery, in providing retro-illumination during phacoemulsification 
because of insufficient red reflex caused by dense vitreous hemorrhage ${ }^{(17)}$. To minimize the surgical complications and maximize the surgical outcomes, intra-vitreal injection of anti- VEGF agents before diabetic PPV has been applied as an adjunctive therapy because it is approved to reduce the duration of surgery, decrease retinal breaks, and lessen intraoperative bleeding and also lead fewer endodiathermy applications (18). However, timing of preoperative anti-VEGF is a controversy particularly in an advanced case of ERM with TRD to achieve the hemostasis with easy ERM peeling ${ }^{(19,20)}$.

The aim of the current work was to evaluate the efficacy, safety and complications of bimanual pars plana vitrectomy (PPV) for management of diabetic epiretinal membrane (ERM) associated with tractional retinal detachment (TRD).

\section{PATIENTS AND METHODS:}

This study included a total of 27 eyes in 23 patients (four patients bilateral) presented with PDR in the form of Epiretinal membrane with TRD, attending at Department of Ophthalmology, Faculty of Medicine, Al-Azhar University Hospital, Damietta, Egypt. Approval of the ethical committee and a written informed consent from all the subjects were obtained.

This study was conducted between January 2015 and Mars 2018.

\section{Pre-operative preparation}

Full history was taking. Ophthalmological clinical examination including: Measurement of best corrected visual acuity (BCVA). Measurement of intra-ocular pressure (IOP). Slit-lamp examination of the anterior segment and Slit lamp bio-microscopy. Fundus photography if there is clear media. B-scan ultrasonography in opaque media. biometry for all phakic cases.

\section{Preoperative Laboratory investigation:}

Fasting blood glucose, 2hour postprandial blood glucose, coagulation profile, liver and kidney function tests were done to exclude ineligible cases.

Operations were done by the same surgeon under general or local anesthesia according the patient age, and fitness. Pre-operative anti-VEGF (Bevacizumab with dose of $2.5 \mathrm{mg}(0.1 \mathrm{ml})$ [Avastin; Genentech, Inc., San Francisco, CA]) was injected under complete a septic condition in the operating theater in selected cases.

\section{Surgical steps:}

Four ports PPV using trocar system ( $23 \mathrm{G}$ system), the sclerotomies was done at $3.5-4 \mathrm{~mm}$ from the corneal limbus (Fig 1).

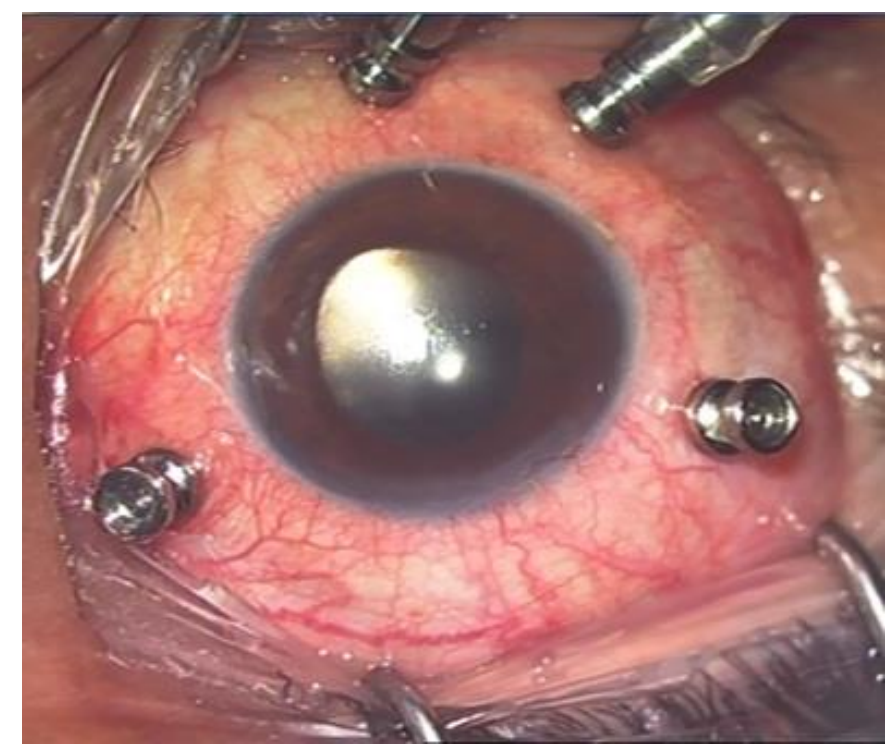

Fig (1): $23 \mathrm{G}$ four ports trocar system, the chandelier trocar $25 \mathrm{G}$ at 6 O'clock.

Visualization was achieved using [OCULUS BIOM®5 or Ziess RESIGHT $\left.{ }^{\circledR} 700\right]$ held on surgical microscope. Core vitrectomy including induction of posterior vitreous detachment (PVD) was done by the ordinary illumination system using parameters; cutting 3500-5000 cuts/minute, vacuum 250-450 $\mathrm{mmHg}$, Triamcinolone Acetonide (TAAc) was used to facilitate visualization (fig 2). Segmentation of the posterior hyaloid membrane was done to remove the adhesions between the retina and the vitreous base before tackling of the ERM. Anterior hyaloid face was then removed through the anterior segment to gain clear media before peeling of ERM.

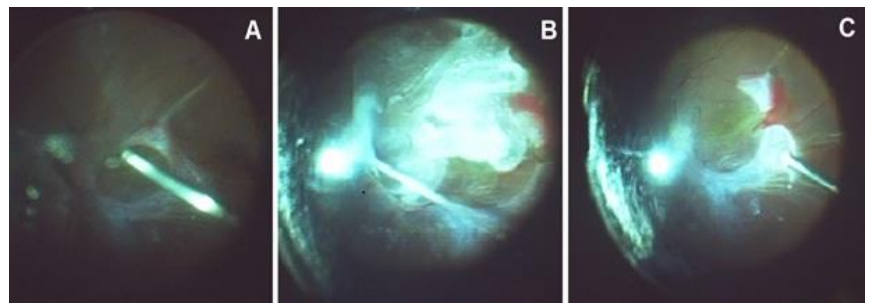

Fig (2): Core vitrectomy and induction of PVD assisted by TAAc injection.

Insertion of the chandelier in the $4^{\text {th }}$ port (fig. 1) was used as a source of illumination with the start of ERM peeling, ERM is then operated bimanually using curved scissors and end-gripping forceps or using cutter and scissors, the assistant maintained direction of the chandelier light toward the scene of the ERM peeling throughout the maneuver to get the maximum illumination by the chandelier system with focus on the area of peeling.

The fibro-vascular membranes were removed by more than one technique, such as membrane dissections, segmentation and en bloc excision. Residual fibrovascular tissue that could not be completely removed 
safely was removed by using the vitreous cutter by trimming to avoid more traction on the retina especially in dangerous areas as around the macula.

De-bridging (Segmentation) of the residual undissectible membranes over the retina is another way to avoid the traction upon the retina which is achieved by cutting of the traction ring using the curved retinal scissors between the adherent points of traction followed by trimming using the vitreous cutter (fig. 3).

Bleeding was controlled by using intraocular diathermy for cauterization of the bleeding point or by using the soft tip back flush simultaneously with suction by the cutter probe with increasing the IOP to avoid excessive use of the diathermy.

Fluid-air exchange and drainage of subretinal fluid through iatrogenic retinal break or retinotomy. Endolaser application was done according to the need. The number of laser shots is determined according to retinal status. The choice of the proper tamponade between Silicone oil, or Sulfur hexafluoride 20\% (SF6, expansile gas) was selected according to the retinal situation at the end of the surgery.
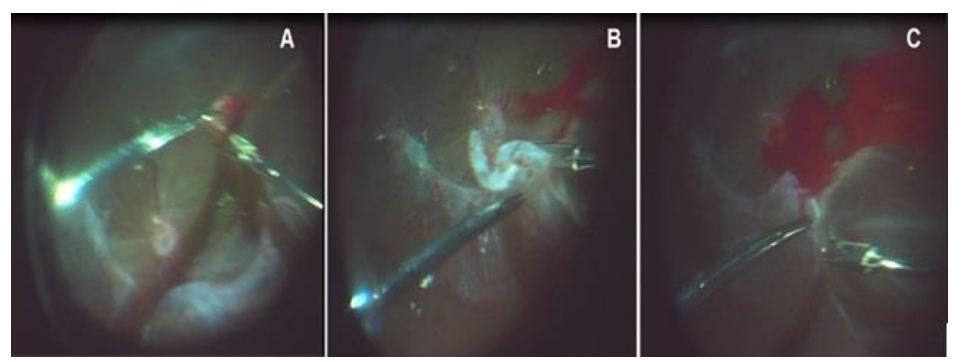

Fig. (3): Bimanual removal of ERM using the chandelier light to be the source of illumination

Combined Phaco-vitrectomy was done, in patient with significant cataract, phacoemulsification was done before PPV if there is good red reflex, in case of dim red reflex due to dense vitreous hemorrhage it was preferred to start with core vitrectomy to gain good reflex or doing with assistance of retroillumination using the chandelier light instead of red reflex. Trypan blue staining of the anterior capsule was used if needed. Suturing of sclerotomies may be done if indicated.

Finally, subconjunctival injection of steroid and antibiotic was done. All patients evaluated 1day post- operative, 1 week, 2 weeks, 1 month and then monthly for 6 months after the surgery. At each follow-up visit full ophthalmological examination was done including: BCVA, IOP, anterior segment and fundus examination.

\section{Statistical analysis}

Data was analyzed using IBM SPSS Advanced Statistics version 20.0 (SPSS Inc., Chicago, IL). Numerical data were expressed as mean and standard deviation. Qualitative data were expressed as frequency and percentage

\section{RESULTS}

All cases diabetic ERM associated with TRD of different degrees (fig.4) in this study underwent bimanual PPV to minimize intra-operative bleeding during ERM peeling, 19 cases of them (70.37\%) were Prepared with Pre-Operative injection with antiVEGF (Bevacizumab 2.5mg/0.1ml) 1-7 days before surgery (Tab.1). Combined phacoemulsification and PPV was done in ten cases $(37.03 \%)$; because of dense vitreous hemorrhage associated with dense cataract; staining of the anterior capsule as well as use of the chandelier retro-illumination was done in phacoemulsification operation. five cases $(18.51 \%)$ were Pseudophakic and eleven cases (40.74 \%) underwent lens sparing PPV due to clear lens or faint cataract not affecting the visualization.

Complete removal of ERM (fig.5) was achieved in 23 cases $(85.18 \%)$ while in four cases $(14.81 \%)$ ERM has incompletely removed; none of them significantly affecting the macular area; Residual TRD was observed in two cases $(7.40 \%)$.

Iatrogenic breaks were unavoidable and occurred but well managed in five cases $(18.51 \%)$, there is no indication for retinectomy. Endo-laser photocoagulation was done in 19 cases $(70.73 \%)$ with minimum about 430 shots and maximum 870 shots, while the remaining 8 cases $(29.62 \%)$ there was no need for intra-operative end-laser.

The choice of the proper tamponade was decided at the end of each case, silicone oil was selected in 24 cases $(88.9 \%)$ while gas was selected in 3 cases $(11.11 \%)$. Bevacizumab was injected at the end of the surgery in 16 cases $(59.25 \%)$ to decrease the vitreous cavity hemorrhage in the early postoperative period and progression of rubeosis.

Table (1) Preoperative data of patients.

\begin{tabular}{|l|l|l|}
\hline \multicolumn{2}{|l|}{ Characteristics } & \\
\hline Age (years) & $19-75$ & \\
\hline Range & 53.9 & \\
\hline Mean &
\end{tabular}




\begin{tabular}{|l|l|l|}
\hline Sex & $\begin{array}{l}11 \text { Eyes } \\
(40.7 \%)\end{array}$ \\
\hline Male \% - Eyes \% & $\begin{array}{l}10 \\
(43.5 \%)\end{array}$ & $\begin{array}{l}16 \text { Eyes } \\
(59.3 \%)\end{array}$ \\
\hline $\begin{array}{l}\text { Female \% - Eyes } \\
\%\end{array}$ & $\begin{array}{l}13 \\
(56.5 \%)\end{array}$ & \\
\hline $\begin{array}{l}\text { Type of DM } \\
\text { (N=23) }\end{array}$ & & $(39.1 \%)$ \\
\hline Type I DM & 9 & $(60.9 \%)$ \\
\hline Type II DM & 14 & \\
\hline Duration of DM (years) & \\
\hline Range & $12-30$ & \\
\hline Mean & 19.2 & \\
\hline Lens status & NO & $\mathbf{\%}$ \\
Phakic & 21 & $(77.77 \%)$ \\
Aphakic & 1 & $(3.70 \%)$ \\
Pseudophakic & 5 & $(18.51 \%)$ \\
\hline Preop anti & NO & $\mathbf{\%}$ \\
VEGF & 19 & $(70.37 \%)$ \\
Yes & 8 & $(29.62 \%)$ \\
No & NO & $\mathbf{\%}$ \\
\hline \multicolumn{2}{|l|}{ Another factors } & $(30.4 \%)$ \\
\hline Hyperlipidemia & 7 & $(52.2 \%)$ \\
Hypertension & 12 & $(26.1 \%)$ \\
Aspirin therapy & 6 & $(8.69 \%)$ \\
Renal failure & 2 & $\mathbf{\%}$ \\
\hline Procedure & NO & $(40.74 \%)$ \\
Lens sparing PPV & 11 & $(22.22 \%)$ \\
PPV & 6 & $(37.03 \%)$ \\
Phaco- PPV & 10 & \\
\hline
\end{tabular}

One case (3.70\%) suffered from hyphemia (grade I) in the first Post-Operative days after combined phacoemulsification- Vitrectomy operation the case managed by medical treatment, Positioning and close follow up of the patient until complete clearance with no need for surgical intervention.

By the end of follow up time, there was improvement of BCVA in $23(85.2 \%)$ cases (Tab.2), while remained the same BCVA in two cases $(7.4 \%)$ and two eyes $(7.4 \%)$ had worsened BCVA. Early and late postoperative complications are summarized in table $(3 \& 4)$.

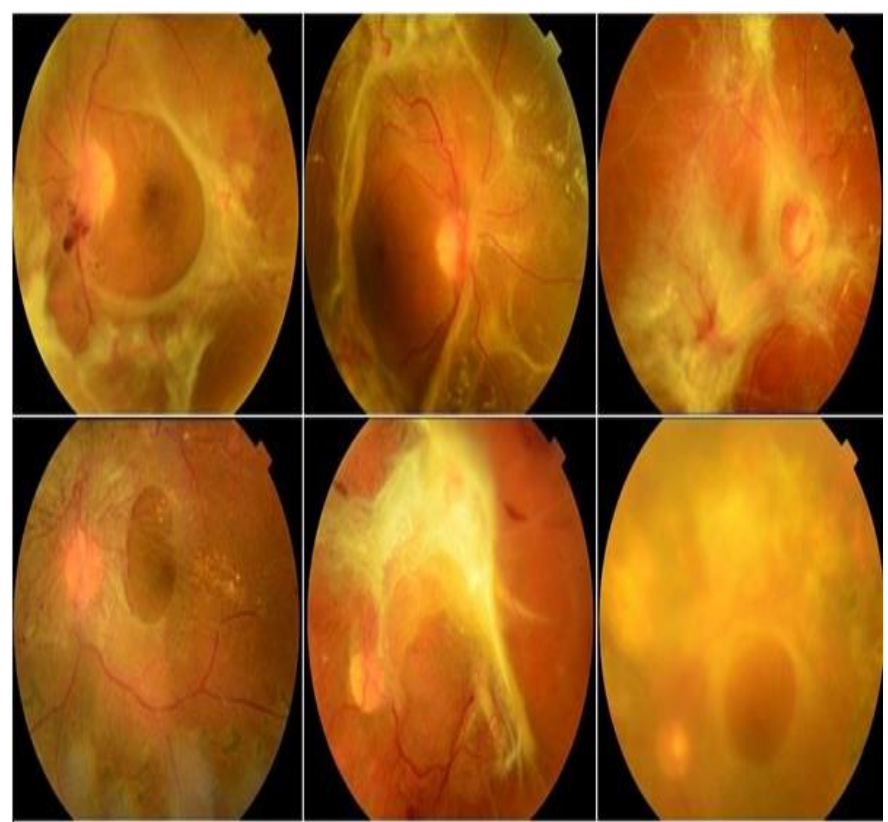

Fig (4): Pre-Operative fundus photo of different cases of diabetic TRD.

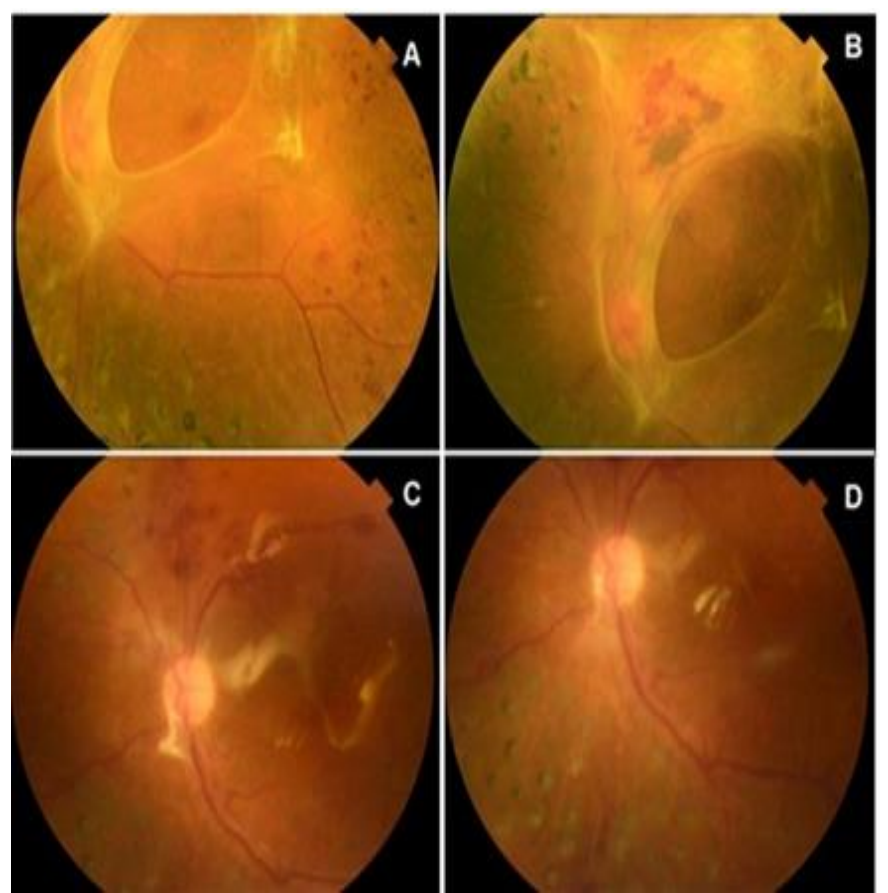

Fig (5): (A\&B) Pre-operative fundus photos of diabetic TRD, (C\&D) Postoperative fundus photos show totally removed ERM, retina totally attached under Silicone oil.

Table (2): The final visual outcome.

\begin{tabular}{|l|l|l|}
\hline $\begin{array}{l}\text { Visual acuity after 6 } \\
\text { months }\end{array}$ & Number & Percentage \\
\hline $\begin{array}{l}\text { Improved by 2 lines } \\
\text { or more }\end{array}$ & 15 & $55.6 \%$ \\
\hline improved by one line & 8 & $29.6 \%$ \\
\hline Remain the same & 2 & $7.4 \%$ \\
\hline Worsened & 2 & $7.4 \%$ \\
\hline
\end{tabular}


Table (3): Early Post-operative complications $\left(1^{\text {st }}\right.$ month).

\begin{tabular}{|l|l|l|}
\hline $\begin{array}{l}\text { Complications } \\
\text { (total=27) }\end{array}$ & No (Cases) & $\mathbf{( \% )}$ \\
\hline $\begin{array}{l}\text { Vitreous cavity } \\
\text { hemorrhage }\end{array}$ & 6 & $22.2 \%$ \\
\hline High IOP & 4 & $14.8 \%$ \\
\hline Corneal edema & 3 & $11.1 \%$ \\
\hline Hyphema & 1 & $3.7 \%$ \\
\hline Residual TRD & 1 & $3.7 \%$ \\
\hline
\end{tabular}

Table (4) Late post-operative complications (after 1 month).

\begin{tabular}{|l|l|l|}
\hline Complications & Number & Percentage \\
\hline $\begin{array}{l}\text { Vitreous cavity } \\
\text { hemorrhage }\end{array}$ & 1 & $3.7 \%$ \\
\hline $\begin{array}{l}\text { Recurrent } \\
\text { proliferation. }\end{array}$ & 2 & $7.4 \%$ \\
\hline Complicated cataract & $5 / 11(\mathrm{LSV})$ & $45.45 \%$ \\
\hline Emulsification of SO & 3 & $11.1 \%$ \\
\hline Secondary glaucoma & 3 & $11.1 \%$ \\
\hline Rubeosis of the Iris & 1 & $3.7 \%$ \\
\hline $\begin{array}{l}\text { Consecutive optic } \\
\text { disc atrophy }\end{array}$ & 2 & $7.4 \%$ \\
\hline Macular edema & 5 & $18.5 \%$ \\
\hline
\end{tabular}

\section{DISCUSSION}

Epiretinal membrane may be associated with superficial or full-thickness retinal folds; resulting in TRD and visual impairment ${ }^{(21)}$. Secondary ERMs are due to another disease either systemic or ocular such as diabetic retinopathy ${ }^{(22)}$. The involvement of cellular proliferation, migration, and adhesion suggest that secondary ERM formation may be an abnormal wound healing and inflammatory response ${ }^{(23)}$. Twenty-seven eyes of twenty-three patients with ERM associated with TRD significantly affecting the vision were included in this study for evaluation of Chandelier assisted Bimanual vitrectomy for diabetic TRD and technique of ERM peeling. Improvement in surgical techniques has improved anatomic and functional success rates following surgery for severe and challenging cases of advanced PDR (22) . A comparative double-blind study between bimanual chandelier assisted PPV versus conventional vitrectomy included sixty patients with diabetic TRD concluded that, the bimanual vitrectomy is helpful for holding forceps to grasp the membranes for separation from the retina using scissors or cutters, bimanual vitrectomy for diabetic cases is safer and effective particular in complicated cases compared with the traditional vitrectomy system. Bimanual vitrectomy had the upper hand as regards the surgical time, better control of intra-operative bleeding, minimal intraoperative diathermy and better final visual outcomes after six months of post-operative follow up (24).

This study discusses the benefits of using bimanual vitrectomy with chandelier endoillumination in advanced cases of ERM associated with TRD as these cases considered challenging because of many difficulties including adherent ERM to the retinal surface with high probability of incidence of iatrogenic retinal tears during ERM dissection, high vascularity of ERM which lead to haziness of the media due to recurrent and excessive intra-operative bleeding which leads to poor visualization in the meticulous step of ERM peeling.

The bimanual vitrectomy has many advantages in such cases as the chandelier gives a good panoramic visualization with less retinal photo-toxicity, the surgeons can use the second hand to deal with the ERM. This technique decreases the traction on the retina, so intraoperative retinal bleeding and breaks will be minimized. The surgeon can use the flute for back flushing of the blood while using the cutter for suction of the blood, decreasing the operative time and post-operative complications. Beside that, bimanual technique also enables the surgeon to do selfindentation during the surgery.

in this study the iatrogenic breaks occur in five cases $(11.6 \%)$ this is compared to $10 \%$ in some studies ${ }^{(25)}, 15 \%$ in other studies ${ }^{(26)}$.

In the current study, PPV was done 1-7 days after intra-vitreal injection of anti VEGF. while some authors suggested variable timing as 1-14 days before surgery ${ }^{(27)}$.

Intra-operative bleeding was significant in eleven cases $(40.74 \%)$, there is statistical evidence of the effect of intra-vitreal preoperative injection of antiVEGF on the intra-operative bleeding, also the need of intra-operative diathermy use was less in the injected group, iatrogenic tears were less in the injected group, surgical time was variable but it was less in the injected group as the recurrent hemorrhage affecting the visualization, leading to haziness of the media, more time to control the bleeding points, more retinal manipulation and instruments exchange with postoperative prolonged recovery period.

The choice of the proper tamponade was determined at the end of vitrectomy in each case according to severity of the case, presence of active intra-operative bleeding, retinal status and retinal breaks.

There is a study reported that nuclear sclerosis after lens-sparing vitrectomy for diabetic retinopathy was less progressive than in other diseases due to the presence of ischemia and low oxygen supply ${ }^{(28)}$. In this study complicated cataract was observed in five of eleven cases $(45.45 \%)$ of lens sparing PPV. Postoperative complications are shown in table (4). 


\section{CONCLUSION}

It could be concluded that bimanual PPV is an effective, safe and an attainable technique for management of diabetic ERM associated with TRD, learning curve with this technique and coast benefit ratio may be considered.

\section{REFERENCES}

1. Klein B (2007): Overview of epidemiologic studies of diabetic retinopathy. Ophthalmic Epidemiol., 14: 179183.

2. Xie X, Xu L, Jonas J (2009): Prevalence of diabetic retinopathy among subjects with known diabetes in China: the Beijing eye study. Eur J Ophthalmol., 19: 9199.

3. Fraser-Bell S, M. Guzowski E, Wang J, Mitchell $\mathbf{P}(\mathbf{2 0 0 3})$ : Five year cumulative incidence and progression of epiretinal membranes: the Blue Mountains Eye Study.Ophthalmology, 110 (1): 34-40.

4. Pournaras C, Donati G, Brzitiko P, Kapetanios A, Dereklis D, Stangos $N$ (2000): Macular epiretinal membranes. Semin Ophthalmol., 15(2):100-115.

5. Hiscott $P$, Hagan $S$, Heathcote L, Sheridan CM, Groenewald CP, Grierson I, Wong D, Paraoan L (2002): Pathobiology of epiretinal and subretinal membranes: possible roles for the matricellular proteins thrombospondin 1and osteonectin (SPARC). Eye, 16 (4): 393-403.

6. Lee S, Pak K, Kwon, Park S, Lee J, Byon I (2018): Association between tangential contraction and early vision loss in idiopathic epiretinal membrane. Retina, 38: 541-549.

7. Massin P, Allouch C, Haouchine B (2000): Optical coherence tomography of idiopathic macular epiretinal membranes before and after surgery. Am J Ophthalmol., 130: 732-739.

8. Niwa T, Terasaki H, Kondo M, Piao C, Miyake Y (2003): Function and morphology of macula before and after removal of idiopathic epiretinal membrane. Ophthalmo Vis Sci., 44: 1652-1656.

9. Hillenkamp J, Saikia P, Gora F (2005): Macular function and morphology after peeling of idiopathic epiretinal membrane with and without the assistance of indocyanine green. Br J Ophthalmol., 89: 437-443.

10.Canan H , Sizmaz S, Altan-Yaycioglu R (2013): Surgical results of combined pars plana vitrectomy and phacoemulsification for vitreous hemorrhage in PDR. Clin Ophthalmol., (8):1597-1601.

11. Reilly G, Melamud A, Lipscomb P, Toussaint B (2015): Surgical outcomes in patients with macular pucker and good preoperative visual acuity after vitrectomy with membrane peeling. Retina, 35: 18171821.

12. Ohji M, Tano Y (1995): Membrane delamination wedge. Arch Ophthalmol., 113: 1335-1336.

13. Eckardt C (2003): Twin lights: a new chandelier illumination for bimanual surgery. Retina, 23 (6): 893894.
14. Hassan T (2014): Advances in vitreous surgery. retina today, (10): 108-110.

15. witmer M , Cahan P (2012): Chandelier lighting during vitreoretinal surgery. retina today, (10): 35-37.

16. Eckardt C, Eckert, Eckardt U (2008): 27-gauge Twinlight chandelier illumination system for bimanual transconjunctival vitrectomy. Retina, 28: 518-519.

17. Jang S, Choi $K$, Lee $S$ (2010): Chandelier retroillumination assisted cataract extraction in eyes with vitreous hemorrhage. Arch Ophthalmol., 128: 911-914.

18. Zhao X, Xia S, Chen Y (2017): Antivascular endothelial growth factor agents pretreatment before vitrectomy for complicated proliferative diabetic retinopathy: a metaanalysis of randomised controlled trials. $\mathrm{Br} J$ Ophthalmol., 102 (8): 1077-1085.

19. Arevalo J, Maia M, Flynn H, Saravia M, Avery R, L. Wu L, Farah M, Pieramici D, Berrocal M, Sanchez J (2008): Tractional retinal detachment following intravitreal bevacizumab (Avastin) in patients with severe proliferative diabetic retinopathy. British Journal of Ophthalmology, 92 (2): 213-216.

20. di Lauro R, De Ruggiero $P$, di Lauro R, di Lauro M, Romano M (2010): Intravitreal bevacizumab for surgical treatment of severe proliferative diabetic retinopathy. Graefe's Archive for Clinical and Experimental Ophthalmology, 248 (6): 785-791.

21. Klein R, Klein B, Wang Q, Moss S (1994): The epidemiology of epiretinal membranes. . Trans Am Ophthalmol Soc., 92: 403-425.

22. Grigorian R, Castellarin A, Fegan R, Seery C, Del Priore L, Von Hagen S, Zarbin M (2003): The prevalence and incidence of epiretinal membranes in eyes with inactive extramacular $\mathrm{CMV}$ retinitis. Invest Ophthalmol Vis Sci., 55: 4304-4312.

23. Stevenson W, Prospero P, Claudia M, Agarwal D, Gelman R, Christoforidis J (2016): Epiretinal membrane: optical coherence tomography-based diagnosis and classification. Clinical ophthalmology , 10: 527-534.

24. Lolah M, Shaarawy A (2014): A comparative study of $23 \mathrm{G}$ vitrectomy and bimanual surgery in the management of epiretinal membranes in diabetic eyes. Journal of Egyptian Ophthalmological Society, 107: 214-219.

25. Dogramaci M, Lee E, Williamson $T$ (2012): The incidence and the risk factors for iatrogenic retinal breaks during pars plana vitrectomy. Eye, 26: 718-722.

26. Ramkissoon Y, Aslam S, Shah, Wong C, Sullivan $P$ (2010): Risk of Iatrogenic Peripheral Retinal Breaks in 20-G Pars Plana Vitrectomy. Ophthalmology, 117:18251830.

27. Ahn J, Woo S, Chung H, Park K (2011): The effect of adjunctive intravitreal bevacizumab for preventing postvitrectomy hemorrhage in proliferative diabetic retinopathy. Ophthalmology, 118: 2218-2226.

28. Holekamp N, Bai F, Shui Y, Almony A, Beebe D (2010): Ischemic diabetic retinopathy may protect against nuclear sclerotic cataract. Am J Ophthalmol., 150: 543550 . 